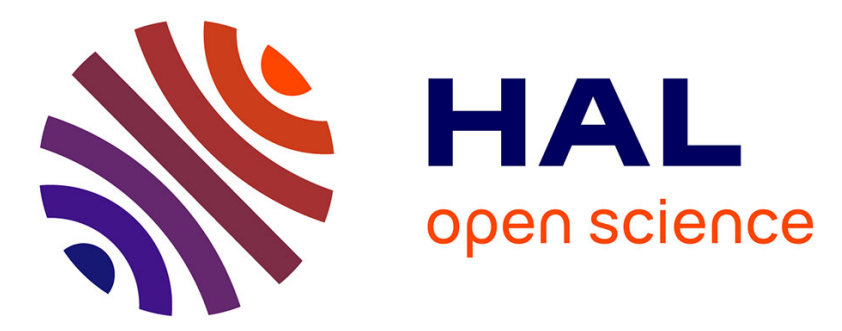

\title{
MULTISCALE FOURTH-ORDER MODEL FOR IMAGE INPAINTING AND LOW-DIMENSIONAL SETS RECOVERY
}

Anis Theljani, Zakaria Belhachmi, Moez Kallel, Maher Moakher

\section{- To cite this version:}

Anis Theljani, Zakaria Belhachmi, Moez Kallel, Maher Moakher. MULTISCALE FOURTH-ORDER MODEL FOR IMAGE INPAINTING AND LOW-DIMENSIONAL SETS RECOVERY. 2016. hal$01114292 \mathrm{v} 3$

\section{HAL Id: hal-01114292 \\ https://hal.science/hal-01114292v3}

Preprint submitted on 9 Feb 2016

HAL is a multi-disciplinary open access archive for the deposit and dissemination of scientific research documents, whether they are published or not. The documents may come from teaching and research institutions in France or abroad, or from public or private research centers.
L'archive ouverte pluridisciplinaire HAL, est destinée au dépôt et à la diffusion de documents scientifiques de niveau recherche, publiés ou non, émanant des établissements d'enseignement et de recherche français ou étrangers, des laboratoires publics ou privés. 


\title{
MULTISCALE FOURTH-ORDER MODEL FOR IMAGE INPAINTING AND LOW-DIMENSIONAL SETS RECOVERY
}

\author{
Anis ThelJANi \\ National Engineering School at Tunis, University of Tunis-El Manar, \\ B.P. 37, 1002 Tunis-Belvédère, Tunisia. \\ ZAKARIA BELHACHMI \\ Mathematics, Information Technology and Applications Laboratory, \\ University of Haute Alsace, France. \\ Moez KalleL \\ IPEIT, Université de Tunis, \\ 2, Rue Jawaher Lel Nehru, 1089 Montfleury, Tunisia. \\ MAHER MOAKHER \\ National Engineering School at Tunis, University of Tunis-El Manar, \\ B.P. 37, 1002 Tunis-Belvédère, Tunisia.
}

(Communicated by the associate editor name)

\begin{abstract}
We consider a fourth-order variational model, to solve the image inpainting problem, with the emphasis on the recovery of low-dimensional sets (edges, corners) and the curvature of the edges. The model permits also to perform simultaneously the restoration (filtering) of the initial image where this one is available. The multiscale character of the model follows from an adaptive selection of the diffusion parameters which allows us to optimise the regularization effects in the neighborhoods of the small features that we aim to preserve. In addition, since the model is based on the high-order derivatives, it favors naturally the accurate capture of the curvature of the edges, hence to balance the tasks of obtaining long curved edges and the obtention of short edges, tip points and corners. We analyze the method in the framework of the calculus of variations and the $\Gamma$-convergence to show that it leads to a convergent algorithm. In particular, we obtain a simple discrete numerical method based on a standard mixed-finite elements with well established approximation properties. We compare the method to the Cahn-Hilliard model for the inpainting and we present several numerical examples to show its performances.
\end{abstract}

1. Introduction. The digital image inpainting started with the works of engineers and computer scientists in the mid-nineties of last century and it refers to the restoration of a scratched or damaged image. In the image processing, this task is very important and has many applications in various fields (painted canvas and movies restoration, augmented reality, ...). Let $\Omega \subset \mathbb{R}^{2}$ denotes the entire image domain and let $D \subset \Omega$ be a damaged part of $\Omega$. The basic idea in the image inpainting is to fill-in $D$, in such a manner that a viewer can not detect the restored part from the rest of the image. Different techniques have been applied to

2010 Mathematics Subject Classification. 65M32, 65M50, 65M22, 94A08, 65N22, 35G15, 35Q68

Key words and phrases. Image inpainting; Inverse problems; Regularization procedures; Mixed finite elements. 
solve such a problem, e.g. some heuristic "copy-paste" procedures [31], statistical approaches [5, 34], we refer to the review article [14] and the references therein for an exhaustive overview on this problem. Among the various methods, the Partial Differential Equations (PDEs) are now widely used and are proven to be efficient in this fields [12, 13, 15, 18, 27, 32, 36, 38, 39]. The underlying idea in the PDE-based methods is to interpolate with a differential operator the data given outside of $D$. A large number of differential operator have been considered, in particular, several second-order diffusion equations have been proposed in the literature [18, 27, 32]. Such operators have the advantage of being well-established theoretically and to lead to various simple numerical methods. However, they may suffer from major drawbacks such as the disconnection of level lines over large distances (violating the connectivity principle), the smooth propagation of the edges into the damaged domain (blurring effect), or the failure in reproducing some high-order features (curvatures, ...). Note also that these methods do not work when the damaged region $D$ touches the boundary of $\Omega$. In such a situation, the authors in [36] proposed an inpainting method based on the data completion with a nonlinear Cauchy problem.

The shortcomings of the second-order partial differential equations gave rise to a class of high-order diffusion models which in general perform better, particularly for restoring the curvatures and for matching edges across large distances. In fact, the supplementary information on the level lines directions which may be provided from the use of boundary conditions both on the solution and its gradient enhance the quality of the reconstruction. In addition, in the homogeneous areas of $\Omega$ the high-order of the operator damps the noise faster than any second order one.

Overview on higher-order PDE models. Bertalmio et al. [15] pioneered a two dimensional fourth-order PDE which consists in propagating both the gradient direction (geometry) and the gray-scale values of the image inside the region to be filled-in by solving the following equation:

$$
\partial_{t} u-\nabla^{\perp} \cdot \nabla \Delta u=0, \text { in } D, u=f, \text { on } \partial D,
$$

where $\nabla^{\perp}$ denotes the perpendicular gradient $\left(-\partial_{y}, \partial_{x}\right)$ and $f$ is the initial damaged image. This model was the subject of other improvements in [13] based on the Navier-Stokes equations. IOther authots [39, 41, 42] considered the Euler's elastica functional to minimize the following energy:

$$
\|u-f\|_{L^{2}(\Omega)}+\int_{K}\left(a+b \kappa^{2}\right) d \mathcal{H}^{1}(x),
$$

where $\mathcal{H}^{1}$ denotes the Hausdorff measure, $K$ is a closed regular subset of $\Omega$ and $\kappa=\nabla \cdot(\nabla u /|\nabla u|)$ is the curvature of the level sets $\gamma_{r}:=\{x \in K \mid u(x)=r\}$ and $a, b$ being two positive constants. In this high-order variational model, the regularization term (Euler's elastica) combines the total variation, sensitive to the length of the isolines, and the square of the curvature, which favors curved lines rather than the straight ones. Minimizing (2) leads to a highly nonlinear PDE and therefore its numerical solution is usually a non trivial task. It was the subject of many numerical investigations $[2,30,32,37]$. In the same spirit, Esedoglu and Shen proposed in [32] the Mumford-Shah-Euler image inpainting model which is a highorder correction of the Mumford-Shah model, incorporating the Willmore energy (integral of the square of the curvature). They propose a numerical scheme based on the $\Gamma$-convergence approximation of Ambrosio and Tortorelli [3, 4]. Another high-order approach was proposed in [17] based on the Cahn-Hilliard equation for 
binary images inpainting. This semi-linear fourth-order PDE originally introduced in material sciences by John W. Cahn and John E. Hilliard in [23] describes the evolution of an interface separating two stable states. A variant named $T V-H^{-1}$ model was proposed in [22] as a generalization to the gray-scale images. In [28, 29], the authors studied the existence of global attractors to some generalized CahnHilliard equations and the effect of the non-linear potential on the model.

The multiscale approach. It ressorts from this overview that most high-order approaches to the inpainting problem lead to the minimization of an energy of type (2). Such models are generally highly nonlinear and require some sophisticated discretizations to be solved numerically. In another hand, the easiest way to obtain a fourth-order PDE is to minimize $\int_{D}|\Delta u|^{2} d x[19,24,43]$ which leads to solve the isotropic fourth-order (stationary) diffusion equation:

$$
\Delta^{2} u=0 \text {, in } D, \quad u=f+\text { another boundary condition on } \partial D \text {. }
$$

Of course, because of its strong smoothing effect, this model cannot capture some relevant features of the image like the corners and the edges which belong to the singular set of the image $f$.

In this article, we consider the following equation:

$$
\begin{cases}\partial_{t} u+\Delta\left(\Delta_{\alpha} u\right)+\lambda_{D}(u-f)=0, & \text { in } \mathbb{R}_{+} \times \Omega, \\ u=\Delta_{\alpha} u=0, & \text { on } \mathbb{R}_{+} \times \partial \Omega, \\ u(0, x)=f, & \text { in } \Omega,\end{cases}
$$

where $\Delta_{\alpha} u=\operatorname{div}(\alpha(x) \nabla u)$ and $\lambda_{D}=\lambda_{0} \chi_{\Omega \backslash D}$ for $\lambda_{0} \gg 0$ and $\chi_{\Omega \backslash D}$ is the indicator function of the sub-domain $\Omega \backslash D$. The values of the diffusion function $\alpha$, which encodes different scales in the image, are dynamically and locally chosen in order to control the "amount" of smoothing of the operator. Note that the homogeneous boundary condition for $u$ is not a restrictive condition and general boundary data can be handled by a lifting operator.

As the model remains linear with respect to $u$, it seems not well suited for capturing fine geometric structures of an image at a first glance. However, we prove that this approach allows us to restore such relevant features. The reason for this, is that the adaptive process which is the key of the method is in fact a nonlinear process, where the values of $\alpha$ are modified during the resolution. Together with a mesh adaption technique, we construct a nonlinear discrete approximation to make the method sensitive to the low-dimensional sets contained in the singular set of $u$. In addition, the process turns out to be $\Gamma$-convergent. Loosely speaking, the method allows us to adjust the model by modifying locally the diffusion coefficients instead of using a priori some nonlinear diffusion law or some nonlinear potential like in the Cahn-Hilliard based equations. This yields -and it is not a small advantagea simple numerical method which consists of solving only linear problems on $u$ and updating the diffusion parameters from the informations given by some error indicators. In addition, we emphasize that a strong point in this approach is that it conciliates a local character of the parameter selection which enhance the accuracy and the global character given by the PDEs framework which ensures the coherence of the reconstruction for large features. Thus, it constitutes, as showed by the numerical simulations a balance between the performances of the local methods, mainly based on "copy-paste" procedures and well suited for textured images, and the performances of global methods (typically PDEs). 
Organization of the paper. The remainder of this article is organized as follows: In Section 2, we prove by standard variational techniques and a fixed point theorem the existence of $H^{1}$-solutions for the image inpainting problem. We obtain our equation as the gradient flow of an energy based on an $H^{-1}$-fidelity term. In Section 3, we describe in details the adaptive strategy. In particular, we show that it is a two-step approach where, a mesh-adaptation based on the metric error indicator is used first to fit the geometry of the computed solution, and second a residual type error indicator is used to locally select the value of $\alpha$. We perform the $\Gamma$-convergence analysis of this process and we show that the solution $u$ generated by the adaptive strategy approximates a solution of a new model which combines the Mumford-Shah functional and the $H^{-1}$-fidelity term. In Section 4, we recall the Cahn-Hilliard equation for image inpainting that we will use for the comparison with our model. Finally, in Section 5 we implement our approach and treat several numerical examples to test its efficiency and robustness.

2. Image inpainting problem. We study in this section the image inpainting problem by considering the system (4). For the sake of clarity, in the rest of the paper we omit the time dependence of functions and we use the notation $u$ instead of $u(t)$.

2.1. $H^{1}$-weak solution of the stationary equation. Assume that $\Omega$ is a bounded open set of $\mathbb{R}^{2}$ with piecewise smooth boundary $\partial \Omega$. We recall that the operator $\Delta^{-1}$ is the inverse of the negative Laplacian with homogeneous Dirichlet boundary conditions, i.e., $u=\Delta^{-1} g$ is the unique solution of:

$$
\begin{cases}-\Delta u=g, & \text { in } \Omega, \\ u=0, & \text { on } \partial \Omega .\end{cases}
$$

Let $H^{-1}(\Omega)$ be the dual space of $H_{0}^{1}(\Omega)$ with corresponding norm $\|u\|_{-1}=\left\|\nabla \Delta^{-1} u\right\|_{2}$ and inner product $\langle u, v\rangle_{-1}=\left\langle\nabla \Delta^{-1} u, \nabla \Delta^{-1} v\right\rangle_{2}$, where $\langle\cdot, \cdot\rangle_{2}$ and $\|\cdot\|_{2}$ are the classical inner product and its corresponding norm in the space $L^{2}(\Omega)$.

We consider the solution of the following stationary problem:

$$
\begin{cases}\Delta\left(\Delta_{\alpha} u\right)+\lambda_{D}(u-f)=0, & \text { in } \Omega, \\ u=\Delta_{\alpha} u=0, & \text { on } \partial \Omega,\end{cases}
$$

which can be seen as stationary solution of (4). Problem (6) can be rewritten as follows:

$$
\begin{cases}\Delta_{\alpha} u+\Delta^{-1}\left(\lambda_{D}(f-u)\right)=0, & \text { in } \Omega, \\ u=\Delta_{\alpha} u=0, & \text { on } \partial \Omega,\end{cases}
$$

or equivalently as a coupled elliptic system:

$$
\begin{cases}-\Delta_{\alpha} u=w, & \text { in } \Omega, \\ -\Delta w=\lambda_{D}(f-u), & \text { in } \Omega, \\ u=w=0, & \text { on } \partial \Omega .\end{cases}
$$

The weak formulation of problem (7) is: Find $u \in H_{0}^{1}(\Omega)$ such that:

$$
\langle\alpha \nabla u, \nabla \phi\rangle_{2}-\left\langle\left(\lambda_{D}(f-u), \phi\right\rangle_{-1}=0, \quad \forall \phi \in H_{0}^{1}(\Omega) .\right.
$$


Throughout the paper, we suppose that he domain $\Omega$ is partitioned into $I$ disjoint sub-domains $\left(\Omega_{\ell}\right)_{\ell}$ such that $\alpha$ is given by the piecewise constant scalar function:

$$
\alpha=\alpha_{\ell}, \text { in } \Omega_{\ell}, \quad \ell=1, \ldots, I .
$$

We denote $\alpha_{m}=\min _{1 \leq \ell \leq I} \alpha_{\ell}>0$ and $\alpha_{M}=\max _{1 \leq \ell \leq I} \alpha_{\ell}$.

Note that even if $f \in L^{2}(\Omega)$, from the elliptic regularity results, one has $w \in$ $H^{2} \cap H_{0}^{1}(\Omega)$ however, the solution $u$ might be only $H_{0}^{1}(\Omega)$ since $\alpha$ is only bounded and measurable. In fact, we recall the following regularity result for the operator $-\operatorname{div}(\alpha \nabla(\cdot)):[9$, Proposition 2.5]

Proposition 1. There exists a constant $c$ depending only on the geometry of $\Omega$, such that a weak solution $u$ of the associated Dirichlet problem belongs to $H^{s+1}(\Omega)$, for all real numbers $s<s_{0}$, where $s_{0}$ is given by:

$$
s_{0}=\min \left\{\frac{1}{2}, c\left|\log \left(1-\frac{\alpha_{m}}{\alpha_{M}}\right)\right|\right\} .
$$

Following the methodology used in [22], we now prove the existence of the $H^{1}$ weak solution of the stationary problem by means of Schauder's fixed-point theorem.

Proposition 2 (Schauder's fixed-point theorem [33]). Let $X$ be a Banach space and $K \subset X$ be a compact and convex set of $X$. Assume that the operator $T: K \longrightarrow K$ is continuous. Then $T$ admits a fixed point.

For $R>0$, to be chosen later, we define the convex set:

$$
V=\left\{u \in H_{0}^{1}(\Omega) ;\left\|u_{2}\right\| \leq R\right\},
$$

and we consider the following minimization problem:

$$
\min _{u \in H_{0}^{1}(\Omega)} \mathcal{J}(u, v),
$$

where

$$
\mathcal{J}(u, v)= \begin{cases}\int_{\Omega} \frac{\alpha(x)}{2}|\nabla u|^{2} d x+\frac{1}{2 \lambda_{0}}\left\|\lambda_{0} u-\lambda_{D} f-\Lambda_{D} v\right\|_{-1}^{2}, & \text { if } u \in V, \\ +\infty, & \text { otherwise }\end{cases}
$$

with $f$ and $v \in L^{2}(\Omega)$ and where we set $\Lambda_{D}=\lambda_{0}-\lambda_{D}$ for brevity.

Proposition 3. Let $v \in L^{2}(\Omega)$, the functional $\mathcal{J}(\cdot, v)$ admits a unique minimizer $u \in V$.

Proof. The functional $\mathcal{J}(\cdot, v)$ is strictly convex. In fact, let $u_{1}$ and $u_{2}$ be two functions in $H_{0}^{1}(\Omega)$ such that $u_{1} \neq u_{2}$ and $\left.t \in\right] 0,1$ [, we have:

$$
\begin{aligned}
t \mathcal{J}\left(u_{1}, v\right)+(1-t) \mathcal{J}\left(u_{2}, v\right)-\mathcal{J}\left(t u_{1}+(1-t) u_{2}, v\right) \\
\quad=\frac{t(1-t)}{2}\left[\int_{\Omega} \alpha(x)\left|\nabla u_{1}\right|^{2} d x+\int_{\Omega} \alpha(x)\left|\nabla u_{2}\right|^{2} d x-2 \int_{\Omega} \alpha(x) \nabla u_{1} \cdot \nabla u_{2} d x\right] \\
\quad+\frac{t(1-t)}{2 \lambda_{0}}\left[\left\|\lambda_{0} u_{1}-\lambda_{D} f-\Lambda_{D} v\right\|_{-1}^{2}+\left\|\lambda_{0} u_{2}-\lambda_{D} f-\Lambda_{D} v\right\|_{-1}^{2}\right] \\
\quad-\frac{t(1-t)}{\lambda_{0}}\left[\int_{\Omega} \nabla \Delta^{-1}\left(\lambda_{0} u_{1}-\lambda_{D} f-\Lambda_{D} v\right) \cdot \nabla \Delta^{-1}\left(\lambda_{0} u_{2}-\lambda_{D} f-\Lambda_{D} v\right) d x\right] \\
\quad=\frac{t(1-t)}{2} \int_{\Omega} \alpha(x)\left|\nabla\left(u_{1}-u_{2}\right)\right|^{2} d x+\frac{t(1-t)}{2 \lambda_{0}}\left\|\lambda_{0}\left(u_{1}-u_{2}\right)\right\|_{-1}^{2}>0 .
\end{aligned}
$$


Furthermore, $\mathcal{J}(\cdot, v)$ is weakly lower semi-continuous in $H^{1}(\Omega)$. We consider a minimizing sequence $\left(u_{n}\right)_{n \in \mathbb{N}}$ of $\mathcal{J}(\cdot, v)$, i.e.,

$$
\mathcal{J}\left(u_{n}, v\right) \underset{n \rightarrow \infty}{\longrightarrow} \inf _{u \in V} \mathcal{J}(u, v)=L .
$$

Then, there is a constant $M>0$ such that $\int_{\Omega} \alpha(x)\left|\nabla u_{n}\right|^{2} d x \leq M$ for all $n \geq 0$, and also we have $\left\|u_{n}\right\|_{2} \leq R$ (otherwise $\mathcal{J}\left(u_{n}, v\right)$ would not be finite). Then, using the boundedness of $\alpha(x)$, we get that the sequence $\left(u_{n}\right)_{n \in \mathbb{N}}$ is uniformly bounded in $H^{1}(\Omega)$. Therefore, there exists a subsequence, still denoted $\left(u_{n}\right)_{n \in \mathbb{N}}$, such that $u_{n} \underset{n \rightarrow \infty}{\rightarrow} u$ weakly in $H^{1}(\Omega)$ and $u_{n} \underset{n \rightarrow \infty}{\longrightarrow} u$ in $L^{2}(\Omega)$, with $\|u\|_{2} \leq R$. Thanks to the continuity of the operator $\Delta^{-1}: H^{-1}(\Omega) \longrightarrow L^{2}(\Omega)$, we get:

$$
\mathcal{J}(u, v) \leq \liminf _{n \rightarrow \infty} \mathcal{J}\left(u_{n}, v\right) .
$$

The limit $u$ is then a minimizer for $\mathcal{J}(\cdot, v)$. The uniqueness is guaranteed by the strict convexity of $\mathcal{J}(\cdot, v)$.

The Euler-Lagrange equation corresponding to (10) reads:

$$
\begin{cases}-\Delta_{\alpha} u-\Delta^{-1}\left(\lambda_{D}(f-u)+\Lambda_{D}(v-u)\right)=0, & \text { in } \Omega, \\ u=\Delta^{-1}\left(\lambda_{D}(f-u)+\Lambda_{D}(v-u)\right)=0, & \text { on } \partial \Omega .\end{cases}
$$

Its weak formulation is: Find $u \in H_{0}^{1}(\Omega)$ such that:

$$
\langle\alpha \nabla u, \nabla \phi\rangle_{2}-\left\langle\left(\lambda_{D}(f-u)+\Lambda_{D}(v-u)\right), \phi\right\rangle_{-1}=0, \quad \forall \phi \in H_{0}^{1}(\Omega) .
$$

Let $T: L^{2}(\Omega) \longrightarrow L^{2}(\Omega)$ be the operator such that $T(v)=u$ where $u$ is the unique solution of (13). Therefore, if there exists a fixed point $u=v$ of the operator, it will be a solution of problem (9).

Proposition 4. The operator $T$ admits a fixed point $u \in V$. Moreover, $u$ is $H^{1}$ weak solution of the equation (6).

Proof. Let $v \in B(0, R)$ (where $B(0, R)$ denotes the ball in $L^{2}(\Omega)$ with center 0 and radius $R$ ). From Proposition 3, the minimization problem (11) admits a unique minimizer $u=T(v)$ in the space $H^{1}(\Omega)$ such that $u \in B(0, R)$. Since the embedding $H^{1}(\Omega) \hookrightarrow L^{2}(\Omega)$ is compact, the operator $T$ then maps $L^{2}(\Omega) \rightarrow K$, where $K$ is a compact subset of $L^{2}(\Omega)$. Thus we have:

$$
T: B(0, R) \longrightarrow B(0, R) \cap K=\tilde{K},
$$

where $\tilde{K}$ is a compact and convex subset of $L^{2}(\Omega)$. To apply Schauder's fixed-point theorem, it remains to prove that $T$ is continuous in $B(0, R)$. Let $\left(v_{k}\right)_{k \geq 0}$ be a sequence which converges to $v \in L^{2}(\Omega)$ and $T\left(v_{k}\right)=u_{k}$. The function $u_{k}$ is then the unique minimizer of (11) associated with $v_{k}$, and we have: $\mathcal{J}\left(u_{k}, v_{k}\right) \leq \mathcal{J}\left(0, v_{k}\right)$, i.e.,

$$
\mathcal{J}\left(u_{k}, v_{k}\right) \leq \frac{1}{2 \lambda_{0}}\left\|\lambda_{D} f+\Lambda_{D} v_{k}\right\|_{-1}^{2} .
$$

Since $L^{2}(\Omega) \hookrightarrow H^{-1}(\Omega)$, we get $\left\|v_{k}\right\|_{-1} \leq C\left\|v_{k}\right\|_{L^{2}(\Omega)} \leq C R$ and also $\left\|\lambda_{D} f\right\|_{-1} \leq$ $C^{\prime}$ for some given constants $C, C^{\prime}>0$. Accordingly, we obtain the following estimate:

$$
\mathcal{J}\left(u_{k}, v_{k}\right) \leq C^{\prime}+C R^{2}
$$

and then $\left(u_{k}\right)_{k \geq 0}$ is uniformly bounded in $H^{1}(\Omega)$. Thus, we can consider a convergent subsequence $u_{k_{j}} \underset{j \rightarrow \infty}{\rightarrow} u \in H^{1}(\Omega)$ and $u_{k_{j}} \underset{j \rightarrow \infty}{\longrightarrow} u$ in $L^{2}(\Omega)$. Hence, the unique 
(weak) solution $T\left(v_{k}\right)=u_{k}$ of:

$$
\begin{cases}-\Delta_{\alpha} u_{k}-\Delta^{-1}\left(\lambda_{D}\left(f-u_{k}\right)+\Lambda_{D}\left(v_{k}-u_{k}\right)\right)=0, & \text { in } \Omega \\ u_{k}=\Delta^{-1}\left(\lambda_{D}\left(f-u_{k}\right)+\Lambda_{D}\left(v_{k}-u_{k}\right)\right)=0, & \text { on } \partial \Omega\end{cases}
$$

weakly converges to the unique weak solution $u$ of (12). From the uniqueness of the solution in $V$, we obtain $u=T(v)$. We then deduce that $T$ is continuous in $L^{2}(\Omega)$ and the existence of a stationary solution $u$ follows from Schauder's fixedpoint theorem. In addition, this solution satisfies (7), or equivalently, is a stationary solution of the problem (4).

Remark 1. The solution $u$ of equation (6) verifies the estimate:

$$
\alpha_{m}\|\Delta u\|_{2}+\frac{\lambda_{0}}{2}\|u\|_{2} \leq \frac{\lambda_{0}}{2}\|f\|_{2} .
$$

Therefore, we may choose $R=\|f\|_{2}$.

2.2. The evolution equation. Now, observe that the previous existence proof of a stationary solution applies to show that the following unbounded operator (in $\left.L^{2}\right)$ :

$$
A(u)=\Delta\left(\Delta_{\alpha} u\right)+\lambda_{D} u,
$$

is maximal. Moreover, we have:

$$
\langle A(u), u\rangle_{2} \geq \sum_{\ell=1}^{I} \alpha_{m}\langle\Delta u, \Delta u\rangle_{L^{2}\left(\Omega_{\ell}\right)}+\left\langle\lambda_{D} u, u\right\rangle_{2} \geq 0,
$$

which means that it is monotone. Thus it follows from the theory of maximal monotone operators [21], that the evolution problem (4) admits a unique solution $u \in L^{2}\left(0, T ; H_{0}^{1}(\Omega) \cap H^{1}\left(0, T ; H^{-1}(\Omega)\right) \cap C\left([0, T] ; L^{2}(\Omega)\right)\right.$.

As in the stationary case, problem (4) can be splitted into two second-order equations by introducing an auxiliary function $w$ such that:

$$
\begin{cases}\partial_{t} u-\Delta w+\lambda_{D}(u-f)=0, & \text { in } \mathbb{R}_{+} \times \Omega, \\ -\Delta_{\alpha} u=w, & \text { in } \mathbb{R}_{+} \times \Omega, \\ u=w=0, & \text { on } \mathbb{R}_{+} \times \partial \Omega, \\ u(0, x)=f(x), & \text { in } \Omega .\end{cases}
$$

The weak formulation reads then:

Find a pair $(u, w) \in L^{2}\left(0, T ; H_{0}^{1}(\Omega) \cap H^{1}\left(0, T ; H^{-1}(\Omega)\right)\right) \times L^{2}\left(0, T ; H_{0}^{1}(\Omega)\right), u(0, x)=$ $f(x)$, such that:

$$
\begin{cases}\left\langle\partial_{t} u, \phi\right\rangle_{2}+\langle\nabla w, \nabla \phi\rangle_{2}+\left\langle\lambda_{D} u, \phi\right\rangle_{2}=\left\langle\lambda_{D} f, \phi\right\rangle_{2}, & \forall \phi \in H_{0}^{1}(\Omega), \\ \langle\alpha \nabla u, \nabla \psi\rangle_{2}-\langle w, \psi\rangle_{2}=0, & \forall \psi \in H_{0}^{1}(\Omega) .\end{cases}
$$

Let $u$ the solution of problem (4). Then, it is easy to verify that the pair $\left(u,-\Delta_{\alpha} u\right)$, is a weak solution of $(15)$. We consider another solution $\left(u_{1}, w_{1}\right) \in H_{0}^{1}(\Omega) \times H_{0}^{1}(\Omega)$ of the system (15), we then have:

$$
\begin{cases}\left\langle\partial_{t}\left(u-u_{1}\right), \phi\right\rangle_{2}+\left\langle\nabla\left(w-w_{1}\right), \nabla \phi\right\rangle_{2}+\left\langle\lambda_{D}\left(u-u_{1}\right), \phi\right\rangle_{2}=0, & \forall \phi \in H_{0}^{1}(\Omega), \\ \left\langle\alpha \nabla\left(u-u_{1}\right), \nabla \psi\right\rangle_{2}-\left\langle\left(w-w_{1}\right), \psi\right\rangle_{2}=0, & \forall \psi \in H_{0}^{1}(\Omega) .\end{cases}
$$


Let $\left(\zeta_{\ell}\right)_{\ell=1}^{I}$ be a partition of unity associated to the decomposition $\left(\Omega_{\ell}\right)_{1}^{I}$, and picking $\psi=\alpha_{\ell}^{-1} \zeta_{\ell}\left(w-w_{1}\right)$, in the second equation, we have the identity:

$$
\int_{\Omega_{\ell}} \alpha_{\ell}^{-1} \zeta_{\ell}\left(w-w_{1}\right)^{2} d x=\int_{\Omega_{\ell}} \alpha_{\ell} \nabla\left(u-u_{1}\right) \alpha_{\ell}^{-1} \nabla \zeta_{\ell}\left(w-w_{1}\right) d x, \quad \forall l=1, \cdots, I .
$$

Summing up, we get (after integrating by parts twice the right-hand side):

$$
\sum_{\ell=1}^{I} \alpha_{\ell}^{-1} \int_{\Omega_{\ell}} \zeta_{\ell}\left(w-w_{1}\right)^{2} d x=\int_{\Omega} \nabla\left(u-u_{1}\right) \nabla\left(w-w_{1}\right) d x \geq 0 .
$$

By choosing the test function $\phi=u-u_{1}$ in the first equation and using (17) and the positivity of $\lambda_{D}$, we obtain:

$$
\left\langle\partial_{t}\left(u-u_{1}\right), u-u_{1}\right\rangle_{2}=-\left\langle\nabla\left(u-u_{1}\right), \nabla\left(w-w_{1}\right)\right\rangle_{2}-\left\langle\lambda_{D}\left(u-u_{1}\right),\left(u-u_{1}\right)\right\rangle_{2} \leq 0 .
$$

So that

$$
\partial_{t}\left(\left\|u(t)-u_{1}(t)\right\|_{2}^{2}\right)=2\left\langle\partial_{t}\left(u-u_{1}\right), u-u_{1}\right\rangle_{2} \leq 0 .
$$

It follows that the function $t \mapsto\left\|u(t)-u_{1}(t)\right\|_{2}^{2}$ is decreasing on $\mathbb{R}_{+}$. Since $u(0)=$ $u_{1}(0)$, we get $u=u_{1}$ which implies that $w=w_{1}$. Thus, problems (4) and (15) are equivalent.

2.3. Semi-discrete time problem. For the discretization of the time derivative $\partial_{t} u$, we use the forward Euler scheme. Let $\frac{u-u^{\text {old }}}{\Delta t}$ be an approximation of $\partial_{t} u$, where $\Delta t$ is a time step, $u^{\text {old }}$ and $u$ are the solutions at time $t_{\text {old }}$ and $t=t_{\text {old }}+\Delta t$, respectively. Therefore, time discretization together with splitting scheme (14) leads to the following time-stepping problem:

$$
\begin{cases}\left\langle\frac{u-u^{\text {old }}}{\Delta t}, \phi\right\rangle_{2}+\langle\nabla w, \nabla \phi\rangle_{2}+\left\langle\lambda_{D} u, \phi\right\rangle_{2}=\left\langle\lambda_{D} f, \phi\right\rangle_{2}, & \forall \phi \in H_{0}^{1}(\Omega), \\ \langle\alpha \nabla u, \nabla \psi\rangle_{2}-\langle w, \psi\rangle_{2}=0, & \forall \psi \in H_{0}^{1}(\Omega) .\end{cases}
$$

Proposition 5. For a fixed $u^{\text {old }} \in H_{0}^{1}(\Omega)$, the problem (18) admits a solution $(u, w) \in H_{0}^{1}(\Omega) \times H_{0}^{1}(\Omega)$.

Proof. For a given $v \in L^{2}(\Omega), u^{\text {old }} \in H_{0}^{1}(\Omega)$ and $\Delta t>0$, we define:

$$
\lambda_{D}^{\prime}=\lambda_{D}+\frac{1}{\Delta t} \text { and } f^{\prime}=\frac{1}{\lambda_{D}^{\prime}}\left(\lambda_{D} f+\frac{u_{\text {old }}}{\Delta t}\right) .
$$

We then consider the following problem:

$$
\begin{cases}-\Delta_{\alpha} u-\Delta^{-1}\left(\lambda_{D}^{\prime}\left(f^{\prime}-u\right)\right)=0, & \text { in } \Omega, \\ u=\Delta_{\alpha} u=0, & \text { on } \partial \Omega .\end{cases}
$$

Similarly to Proposition 3, we can prove the existence of $H^{1}$ - solution of (19) such that the pair $\left(u,-\Delta_{\alpha} u\right)$ satisfies the system (18). To prove the uniqueness, let $\left(u_{1}, w_{1}\right) \in H_{0}^{1}(\Omega) \times H_{0}^{1}(\Omega)$ be another solution of the system (18), we then have:

$$
\begin{cases}\left\langle\nabla\left(w-w_{1}\right), \nabla \phi\right\rangle_{2}+\left\langle\lambda_{D}^{\prime}\left(u-u_{1}\right), \phi\right\rangle_{2}=0, & \forall \phi \in H_{0}^{1}(\Omega), \\ \left\langle\alpha \nabla\left(u-u_{1}\right), \nabla \psi\right\rangle_{2}-\left\langle\left(w-w_{1}\right), \psi\right\rangle_{2}=0, & \forall \psi \in H_{0}^{1}(\Omega) .\end{cases}
$$

Following the same lines than for the system (15) and using the inequality (17), we obtain:

$$
\left\langle\lambda_{D}^{\prime}\left(u-u_{1}\right),\left(u-u_{1}\right)\right\rangle_{2}=-\left\langle\nabla\left(u-u_{1}\right), \nabla\left(w-w_{1}\right)\right\rangle_{2} \leq 0 .
$$


From the nonnegativity of $\lambda_{D}^{\prime}$, we get:

$$
\left\langle\lambda_{D}^{\prime}\left(u-u_{1}\right),\left(u-u_{1}\right)\right\rangle_{2}=0 .
$$

Therefore $u=u_{1}$ and consequently $w=w_{1}$.

3. Fully discrete problem and the adaptive strategy. We assume that the domain $\Omega$ is polygonal and we consider a regular family of triangulations $\mathbb{T}_{h}$ made of element which are triangles (or quadrilaterals) with a maximum size $h$, satisfying the usual admissibility assumptions, i.e., the intersection of two different elements is either empty, a vertex, or a whole edge. For $h>0$, we introduce the following discrete space:

$$
X_{h}=\left\{v_{h} \in C(\bar{\Omega})\left|\forall K \in \mathbb{T}_{h}, v_{h}\right|_{K} \in P_{1}(K)\right\} \cap H_{0}^{1}(\Omega) .
$$

The discretized version of the splitted problem (15) leads to finding a pair $\left(u_{h}, w_{h}\right) \in X_{h} \times X_{h}$ solution of:

$$
\begin{cases}\left\langle\frac{u_{h}-u_{h}^{\text {old }}}{\Delta t}, \phi_{h}\right\rangle_{2}+\left\langle\nabla w_{h}, \nabla \phi_{h}\right\rangle_{2}+\left\langle\lambda_{D} u_{h}, \phi_{h}\right\rangle_{2}=\left\langle\lambda_{D} f_{h}, \phi_{h}\right\rangle_{2}, & \forall \phi_{h} \in X_{h}, \\ \left\langle\alpha \nabla u_{h}, \nabla \psi_{h}\right\rangle_{2}-\left\langle w_{h}, \psi_{h}\right\rangle_{2}=0, & \forall \psi_{h} \in X_{h},\end{cases}
$$

where $f_{h}$ is a finite element approximation of $f$ associated with $\mathbb{T}_{h}$. Since $X_{h} \subset$ $H_{0}^{1}(\Omega)$, the well-posedness of the problem (20) follows from (18).

3.1. $\Gamma$-convergence analysis and the adaptive algorithm. In this section, we will prove that the approach considered in the article allows us to approximate, in the $\Gamma$-convergence sense, a new model that couples a Mumford-Shah functional with an $H^{-1}$-term, which we will call MS- $H^{-1}$.

$\Gamma$-convergence analysis. A $\Gamma$-convergence study of this adaptive strategy was presented in [11] for optic flow estimation. The authors proved that this algorithm is equivalent to the adaptive one introduced by Chambolle-Dal Maso [26] and Chambolle-Bourdin [25] where a numerical discrete approximation of the MumfordShah energy was proposed. This approach, based on finite-element discretization and adaptive mesh strategy, is a good approximation, in the $\Gamma$-convergence sense [20] of the Mumford-Shah energy [40] (see [26] for more details). We briefly recall the results and the numerical approximation of this method. For a fixed angle $0<\theta_{0} \leq 2 \pi / 3$, a constant $c \geq 6$, and for $\epsilon>0$, let $\mathcal{T}_{\epsilon}(\Omega)=\mathcal{T}_{\epsilon}\left(\Omega ; \theta_{0} ; c\right)$ be the set of all triangulations of $\Omega$ whose triangles $K$ have the following characteristics:

(i) The length of each of the three edges of $K$ is between $\epsilon$ and $\epsilon c$.

(ii) The three angles of $K$ are greater than or equal to $\theta_{0}$.

Let $V_{\epsilon}(\Omega)$ be the set of all continuous functions $u: \Omega \longrightarrow \mathbb{R}$ such that $u$ is affine on each triangle $K$ of a triangulation $\mathbb{T} \in \mathcal{T}_{\epsilon}(\Omega)$. For a given $u$, we define $\mathcal{T}_{\epsilon}(u) \subset \mathcal{T}_{\epsilon}(\Omega)$ as the set of all triangulations adapted to the function $u$, i.e., such that $u$ is piecewise affine on $\mathbb{T}$. We consider a non-decreasing continuous function $g:[0,+\infty) \longrightarrow$ $[0,+\infty)$ such that:

$$
\lim _{t \rightarrow 0} \frac{g(t)}{t}=1, \lim _{t \rightarrow+\infty} g(t)=g_{\infty}<+\infty .
$$

For any $u \in L^{p}(\Omega),(p \geq 1)$ and $\mathbb{T} \in \mathcal{T}_{\epsilon}(\Omega)$, the authors in [26] introduced the following minimization problem:

$$
G_{\epsilon}(u)=\min _{\mathbb{T} \in \mathcal{T}_{\epsilon}(\Omega)} \tilde{G}_{\epsilon}(u, \mathbb{T}),
$$


where

$$
\tilde{G}_{\epsilon}(u, \mathbb{T})= \begin{cases}\sum_{K \in \mathbb{T}}|K \cap \Omega| \frac{1}{h_{K}} g\left(h_{K}|\nabla u|^{2}\right), & u \in V_{\epsilon}(\Omega), \mathbb{T} \in \mathcal{T}_{\epsilon}(\Omega), \\ +\infty, & \text { otherwise. }\end{cases}
$$

When $\epsilon$ goes to zero and provided $\theta_{0}$ is less than some $\Theta>0$, it was proven that the energy $G_{\epsilon} \Gamma$-converges to the Mumford-Shah functional:

$$
G(u)= \begin{cases}\int_{\Omega}|\nabla u(x)|^{2} d x+g_{\infty} \mathcal{H}^{1}\left(S_{u}\right), & u \in L^{2}(\Omega) \cap G S B V(\Omega), \\ +\infty, & u \in L^{2}(\Omega) \backslash G S B V(\Omega),\end{cases}
$$

where is $G S B V(\Omega)$ the generalized special function of bounded variation (see [1]).

Remark 2. If $F: X \longrightarrow[-\infty,+\infty]$ is continuous and $\left(G_{\epsilon}\right)_{\epsilon} \Gamma$-converges to $G$ then $\left(F+G_{\epsilon}\right)_{\epsilon} \Gamma$-converges to $F+G$.

From the result of the $\Gamma$-convergence of $G_{\epsilon}$ to $G$, see [26, Theorem 2], the continuity of the second term of the functional in $L^{2}(\Omega)$ (which follows from the continuity of $\Delta^{-1}$, i.e., the stability in the elliptic problems) and Remark 2, we have:

Proposition 6. Let $f$ and $v$ in $L^{2}(\Omega)$ be two given functions and $\epsilon>0$ be a positive parameter. Therefore, the sequence of functionals

$$
G_{\epsilon}\left(u_{\epsilon}\right)+\frac{1}{2 \lambda_{0}}\left\|\lambda_{0} u_{\epsilon}-\lambda_{D} f-\left(\lambda_{0}-\lambda_{D}\right) v\right\|_{-1}^{2},
$$

$\Gamma$-converges for $\epsilon \longrightarrow 0$ in the topology of $L^{2}(\Omega)$ to

$$
G(u)+\frac{1}{2 \lambda_{0}}\left\|\lambda_{0} u-\lambda_{D} f-\left(\lambda_{0}-\lambda_{D}\right) v\right\|_{-1}^{2} .
$$

In the theorem we note that the variable $\alpha$ do not appear explicitly in $G$ or $G_{\epsilon}$. To introduce such an $\alpha$, let $\psi$ be the Legendre-Fenchel transform of $g$. For a given triangulation $\mathbb{T}_{\epsilon}$, it was proven in [26] that the minimization of $G_{\epsilon}$ is equivalent to the minimization of the following functional:

$$
G_{\epsilon}^{\prime}\left(u, v, \mathbb{T}_{\epsilon}\right)=\sum_{K \in \mathbb{T}_{\epsilon}}|K \cap \Omega| \frac{1}{h_{K}}\left(v_{K}|\nabla u|^{2}+\frac{\psi\left(v_{K}\right)}{h_{K}}\right),
$$

over all $u \in V_{\epsilon}(\Omega)$ and $v=\left(v_{K}\right)_{K \in \mathcal{T}_{\epsilon}}(\Omega)$, piecewise constant on each $K \in \mathcal{T}_{\epsilon}$. For a fixed $u$, it follows from standard convex analysis that the minimizer over each $v$ is explicitly given by:

$$
v_{K}=g^{\prime}\left(h_{K}|\nabla u|^{2}\right) .
$$

Choosing $\alpha=v$ leads to the adaptive strategy that we describe now..

Remark 3. The analysis presented here is carried out with the Neumann boundary conditions on $u$, which is the framework used in $[11,25,26]$ for a denoising problem or optic flow estimation. The application to the Dirichlet case requires some (tedious but non-essential) modifications and the result still holds.

3.2. Adaptive procedure. For each element $K \in \mathbb{T}_{h}$, the following local discrete energy:

$$
\eta_{K}=\alpha_{K}^{\frac{1}{2}}\left\|\nabla u_{h}\right\|_{L^{2}(K)},
$$

contains some information on the error distribution of the computed solution $u_{h}$. In fact, the discontinuities (edges) are contained in regions where the brightness changes sharply and consequently where this error indicator is large. Moreover, it 
may be proven that the gradient of $u_{h}$ captures this change in brightness and its magnitude provides an information about the "strength" of the edges (see [10]). Thus, the quantity (23) acts as an edge detector and locates such regions. Furthermore, this local error indicator is in some sense equivalent to the energy norm mostly used as an edge detection in the topological gradient based-methods $[6,7,8]$. This particularity makes it well suited to control and locally select the diffusion coefficient $\alpha$ using the following algorithm:

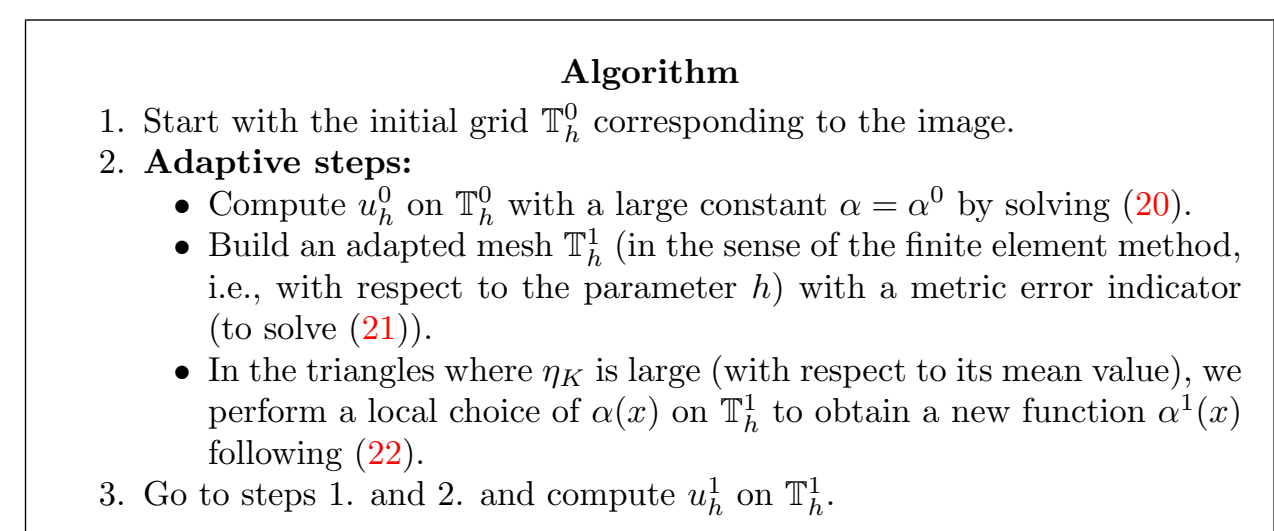

We emphasize that we have to solve only linear problems on $u$ and then to update $\alpha$. Actually, in place of introducing a function $g$ like in the theorem, we use the following formula for the update of $\alpha$ in each triangle $K$ :

$$
\alpha_{K}^{k+1}=\max \left(\frac{\alpha_{K}^{k}}{1+\tau *\left(\left(\frac{\eta_{K}}{\|\eta\|_{\infty}}\right)-0.1\right)^{+}}, \alpha_{t r h}\right),
$$

where $\alpha_{t r h}$ is a threshold parameter and $\tau$ is a coefficient which controls the rate of decrease in $\alpha, u^{+}=\max (u, 0)$. Here $\eta$ is the piecewise-constant function such that $\left.\eta\right|_{K}=\eta_{k}, \forall K \in \mathbb{T}_{h}^{1}$

Remark 4. There are other several possibles choices of functions $g$ to compute $\alpha$. The formula (24) corresponds to $\min \left(t, g_{\infty}\right)$ and is the one we implemented for the numerical computations here. Loosely speaking, it may be understood as follows: in regions of high gradients, it decreases the values of $\alpha$ when the error indicator deviates more then $10 \%$ from its mean value. $\alpha$ decreases nearly as a geometric sequence with the iteration number, until a given threshold is attained.

Let us give more details on the implementation of this algorithm. First, we build an adapted mesh $\mathbb{T}_{h}^{1}$ as follows: close to the jump sets of $u_{h}$, the error is large, we then cut the element $K$ into a finite number of smaller elements to decrease such an error and to fit the edges, while, far from these jump sets, there is no restriction on how to choose the triangles and the initial grid is coarsened. The adopted meshes have small number of degrees of freedom in the homogeneous regions which makes the method considerably fast. Second, we perform an "optimal" choice of the function $\alpha$ following the map furnished by the error indicator $\left(\eta_{K}\right)_{K \in \mathbb{T}_{h}}$, on each element $K$ and in accordance with (24) in order to correctly approximate edges. 
To conclude this section let us try to interpret in term of a PDE the $\Gamma$-limit $G$. We may say that the sequence $\left(u_{\epsilon}, \alpha_{\epsilon}\right)$ constructed by the adaptive algorithm is $\Gamma$-convergent to a Mumford-Shah- $H^{-1}$ functional $\left(\mathrm{MS}-H^{-1}\right)$ and that it solves

$$
\Delta\left(\Delta_{\alpha_{\epsilon}} u_{\epsilon}\right)+\lambda_{D}\left(u_{\epsilon}-f\right)=0, \text { in } \Omega,
$$

after eliminating $w_{\epsilon}$ from the mixed formulation (20). Of course there are others $\Gamma$-convergent approximation functionals to the MS- $H^{-1}$, e.g. The Cahn-Hilliard model.

4. Cahn-Hilliard equation. For the sake of completeness, we will make a comparison with the Cahn-Hilliard model $[16,17,18]$ that we now recall. The CahnHilliard equation [23] originally refers to John W. Cahn and John E. Hilliard [23] and was introduced to phenomenologically describe phase separation, i.e., the change from one state to another. It is a fourth-order semi-linear PDE and is obtained as the $H^{-1}$-gradient flow of the following Ginzburg-Landau energy:

$$
\frac{1}{2} \int_{\Omega}|\nabla u|^{2} d x+\frac{1}{\epsilon^{2}} W(u),
$$

where phase separation is modeled by the smooth free energy, e.g., double-well potentials $W$ such that:

$$
W(u)=\left(1-u^{2}\right) u^{2},
$$

or a non-smooth free energy as follows:

$$
W(u)=\psi(u)+I_{[0,1]}(u),
$$

where $\psi(u)=\frac{1}{2} u(1-u)$, and $I_{[0,1]}(u):= \begin{cases}\frac{1}{2} u(1-u), & 0 \leq u \leq 1, \\ +\infty, & \text { otherwise. }\end{cases}$

The analogy between the Cahn-Hilliard model in material sciences and CahnHilliard model in image inpainting is that the two state phases are considered as two homogeneous regions and the interface is considered as an edge. In image inpainting, it was exploited by Bertozzi et al. in [17] and afterwards in [16, 18, 22] by considering the following equation:

$$
\begin{cases}\partial_{t} u+\Delta\left(\epsilon \Delta u-\frac{1}{\epsilon} W^{\prime}(u)\right)+\lambda_{D}(u-f)=0, & \text { in } \mathbb{R}^{+} \times \Omega, \\ u=f \text { and } \epsilon \Delta u-\frac{1}{\epsilon} W^{\prime}(u)=0, & \text { on } \mathbb{R}^{+} \times \partial \Omega, \\ u(0, x)=f, & \text { in } \Omega,\end{cases}
$$

which was obtained by incorporating the data fidelity term $\lambda_{D}(f-u)$. Note that the classical Chan Hilliard equation (28) is only appropriate for two-scale (binary) images inpainting due to the double well potential $W$ which vanishes on only the values 0 and 1 .

Remark 5. For the time discretization of Cahn-Hilliard equation, we use a convexity splitting scheme (see $[18,22]$ ). The idea is to divide the energy functional into two parts; a convex part plus a concave one. The convex part is then treated implicitly while the concave part is treated explicitly. 
5. Numerical experiments. In this work, all the PDEs are solved with the open source software FreeFem ++ [35]. In all examples, the damaged/missed regions are delimited by the red contour. We give some examples for the application of our proposed approach to image inpainting. The goal is to reconstruct the missing information in the red parts, i.e., $D$, by the diffusion of the information from the intact part, i.e, $\Omega \backslash D$. In all examples, we set $\lambda_{0}=10^{7}$, and $\Delta t=0.1$ and an initial guess $u(0, x)=0.5, \forall x \in D$.

Curvature inpainting. We present the numerical results for the adaptive inpainting approach in order to illustrate the effectiveness of the proposed algorithm for the inpainting of curvature. In Fig. 1, we present the reconstruction of quarter of a circle. We display the evolution of the restored image for iterations 1,5 and 20. In the first iteration ( $\alpha$ is constant), we solved a biharmonic equation which gives a curved, but a very smooth (blurred), edge in $D$. By iterations, we can see the efficiency of the adaptation process in the damaged region where the edge was inpainted sharply by simultaneously keeping its curvature.

Other examples are presented in Fig. 2 and Fig. 4. The curvature in Fig. 2 is well inpainted which proves that our approach, based on fourth-order linear diffusion model, allows us to obtain a very interesting result that one might expect by solving comlpex PDEs like the Euler's elastica [42], which is highly nonlinear and numerically difficult to solve. We give in Fig. 5 a zoom caption in the damaged region 2 which proves that the missing part is well restored and is very close to the original one.

"Real world"-image inpainting. The experiments in Fig. 6 show the efficiency of the proposed method in a real image inpainting. The portions of unknown pixels are $45 \%, 55 \%$ and $75 \%$, respectively. From these experiments, we can see that the proposed model can successfully recover the inpainting domain even when up to $75 \%$ of pixels are unknown.

Comparison with the Cahn-Hilliard model. In Fig. 7, we have chosen the same image presented by Bertozzi, Esedoglu and Gillette (see [17]). We give the result obtained by solving the Cahn-Hiliard equation and the one obtained using our approach. We display the evolution of the restored image at iteration 1, 5 and 10 which show that the edges are progressively and sharply approximated and the four corners are very accurately matched. Notice that the image for Cahn-Hilliard equation is computed in a two-steps process. In the first step, the authors solved their equation with a large value of $\epsilon$, e.g., $\epsilon=0.1$, until the numerical scheme is close to a steady state. In this step, the level lines are continued into the missing domain. In a second step, they used the previous result as an initial time condition $u_{0}$ for a smaller $\epsilon$ (e.g., $\epsilon=0.01$ ) in order to sharpen the contours. This is an adaptive choice for $\epsilon$, however, subjected to a hand tuning and being uniform in the entire domain.

Broken three bars. In Fig. 8, we show the inpainting result for a synthetic image which represents three broken bars forming a $T$-junction. We give the restored images using total variation, harmonic and biharmonic models, respectively. As excepted, total variation inpainting model is unable to connect the edges between the three bars because it does not fulfill the connectivity principle. Harmonic and biharmonic inpainting models produce a smooth solution $u$ in $D$, blurring the edges. However, the proposed approach is able to sharply connect the edges between three bars while enforcing the curvature. 
6. Conclusion. In this article, we have investigated a multiscale approach to a diffusion operator of fourth-order for the image inpainting. The model is linear with respect to the variable $u$ representing the image to be reconstructed and is depending on a spatially varying diffusion function. We introduced an adaptive approximation procedure based on some a posteriori error indicators, which allows us to select the diffusion coefficient using the available data and the informations on the computed solution. We analyzed this approach from the variational point of view and we established its connections with a Mumford-Shah-like energy, in the sense of the $\Gamma$-convergence. We have implemented the considered model to test the method, we have also made some comparisons with existing approaches to demonstrate its capabilities. We have underlined, in the presented tests, the good quality in the recovery of low-dimensional sets (edges, corners) and of the curvature (of edges) in the inpainted zones. We emphasize that in our approach the adaptive selection of the diffusion coefficients is:

(i) Fully automatic, i.e., no external intervention on the algorithm is required.

(ii) Objective and a posteriori, i.e., the scale of $\alpha$ (the rate of decreasing its values) is explicitly obtained from computable quantities (error indicators) which are sensitive to the singularities of the solution. Thus, no a priori guess is necessary

(iii) Local, i.e., made at each location in the computation domain (finite-element cell, thus the pixel scale).

(iv) Low-cost, in the sens of the number of degrees of freedom, thanks to the coarsening of the mesh in the homogeneous parts of the domain.

In addition, the method may be improved straightforwardly by considering $\alpha$, the diffusion function, as a matrix which introduces some anisotropy in the models and the overall approach is easy to implement in the framework of variational methods of approximation.

\section{REFERENCES}

[1] L. Ambrosio, N. Fusco and D. Pallara, Functions of Bounded Variation and Free Discontinuity Problems, Oxford Mathematical Monographs, 2000.

[2] L. Ambrosio and S. Masnou, A direct variational approach to a problem arising in image reconstruction, Interfaces and Free Boundaries, 5 (2003), 63-81.

[3] L. Ambrosio and M. Tortorelli, Approximation of functional depending on jumps by elliptic functional via $\gamma$-convergence, Comm. Pure Appl. Math., 43 (1990), 999-1036.

[4] L. Ambrosio and M. Tortorelli, On the approximation of free discontinuity problems, Boll. Un. Mat. Ital., 6 (1992), 105-123.

[5] S. Armstrong, A. Kokaram and J. W. Rayner, Nonlinear interpolation of missing data using min-max functions, IEEE Int. Conf. Nonlinear Signal and Image, $\mathbf{7}$.

[6] D. Auroux, L. J. Belaid and B. Rjaibi, Application of the topological gradient method to color image restoration, SIAM Journal on Imaging Sciences, 3 (2010), 153-175.

[7] D. Auroux, L.-D. Cohen and M. Masmoudi, Contour detection and completion for inpainting and segmentation based on topological gradient and fast marching algorithms, Journal of Biomedical Imaging, vol. 2011.

[8] D. Auroux and M. Masmoudi, Image processing by topological asymptotic expansion, Journal of Mathematical Imaging and Vision, 33 (2009), 122-134.

[9] Z. Belhachmi, C. Bernardi and A. Karageorghis, Mortar spectral element discretization of nonhomogeneous and anisotropic Laplace and Darcy equations, M2AN, 41 (2007), 801-824.

[10] Z. Belhachmi and F. Hecht, Control of the effects of regularization on variational optic flow computations, Journal of Mathematical Imaging and Vision, 40 (2011), 1-19.

[11] Z. Belhachmi and F. Hecht, An adaptive approach for segmentation and TV edgeenhancement in the optic flow estimation, Journal of Mathematical Imaging and Vision, $1-20$. 


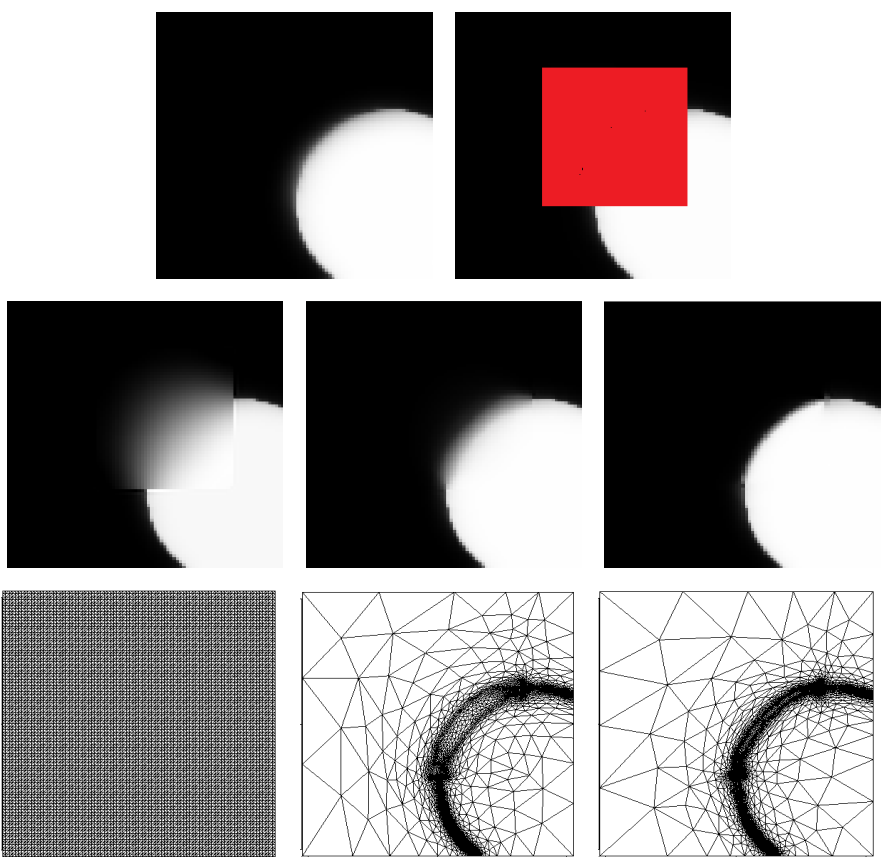

Figure 1. Top row: Original and damaged images. Middle row: Restored image using the model (4) and adaptation at iterations 1, 5 and 20, respectively. Bottom row: Mesh evolution at iterations 1,5 and 20 , respectively.
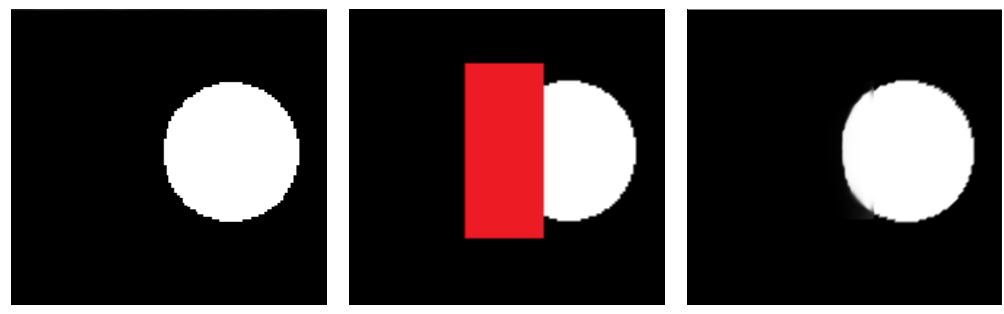

Figure 2. Form left to right: Original, damaged and restored images using model (4) and adaptation.
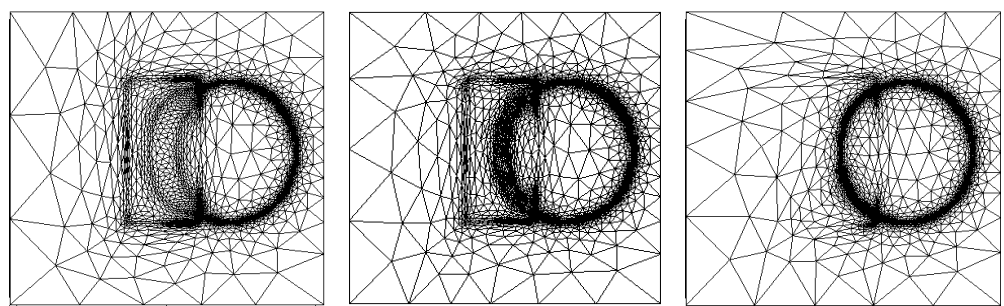

Figure 3. Mesh evolution at iterations 2, 5 and 10, respectively. 

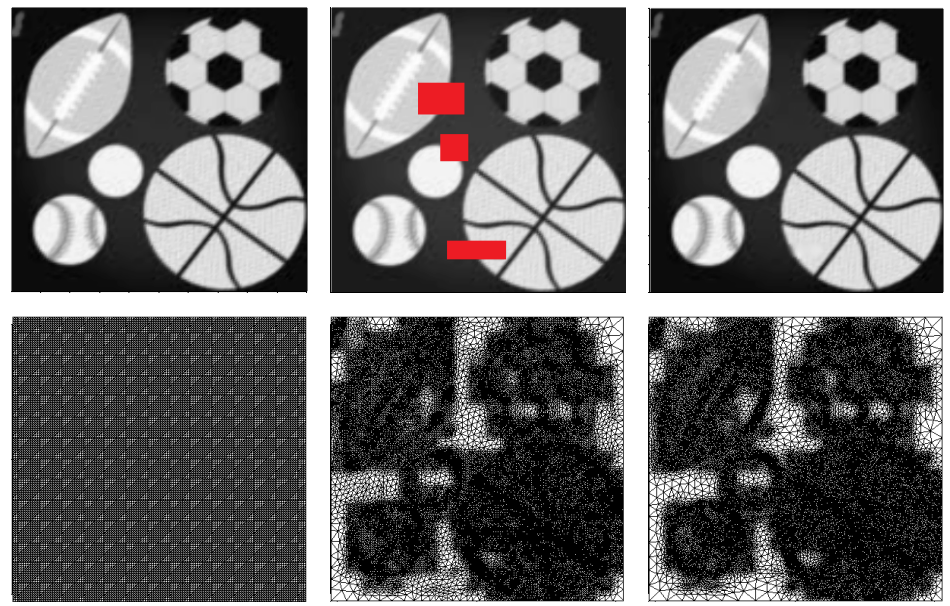

FIguRE 4. Top row: Original, damaged and restored images using model (4) and adaptation., respectively. Bottom row: The evolution of the mesh at iterations 1,5 and 20 , respectively.

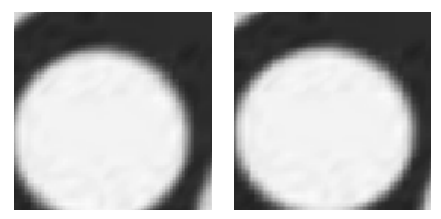

FiguRE 5. Zoom on the damaged region 2: Original and restored.

[12] Z. Belhachmi, M. Kallel, M. Moakher and A. Theljani, Weighted harmonic and complex Ginzburg-Landau equations for gray value image inpainting, International Journal of $\mathrm{Nu}$ merical Analysis and Modeling (2015).

[13] M. Bertalmio, A. L. Bertozzi and G. Sapiro, Navier-Stokes, fluid dynamics, and image and video inpainting, in Proc. IEEE Computer Vision and Pattern Recognition (CVPR), 2001, $355-362$.

[14] M. Bertalmio, V. Caselles, S. Masnou and G. Sapiro, Inpainting, Encyclopedia of Computer Vision, Springer, 2011.

[15] M. Bertalmio, G. Sapiro, V. Caselles and C. Ballesteri, Image inpainting, in Proceedings of the 27th Annual Conference on Computer Graphics and Interactive Techniques, ACM, New York, 2000, 417-427.

[16] A. Bertozzi, S. Esedoglu and A. Gillette, Analysis of a two-scale Cahn-Hilliard model for image inpainting, Multiscale Modeling and Simulation, 6 (2007), 913-936.

[17] A. Bertozzi, S. Esedoglu and A. Gillette, Inpainting of binary images using the Cahn-Hilliard equation, IEEE Trans. Image Proc., 16 (2007), 285-291.

[18] A. Bertozzi and C.-B. Schonlieb, Unconditionally stable schemes for higher order inpainting, Communications in Mathematical Sciences, 9 (2011), 413-457.

[19] A. Blake and A. Zisserman, Visual Reconstruction, MIT Press, Cambridge, MA, USA, 1987.

[20] A. Braides, Gamma-Convergence for Beginners, vol. 22 of Oxford Lecture Series in Mathematics and its Applications, Oxford University Press, 2002.

[21] H. Brézis, Opérateurs Maximaux Monotones et Semi-Groupes de Contractions dans les Espaces de Hilbert, North-Holland Mathematics Studies, North Holland New-York, Amsterdam, London, 1973.

[22] M. Burger, L. He and C.-B. Schnlieb, Cahn-Hilliard inpainting and a generalization for grayvalue images, SIAM J. Imaging Sci., 2 (2009), 1129-1167. 

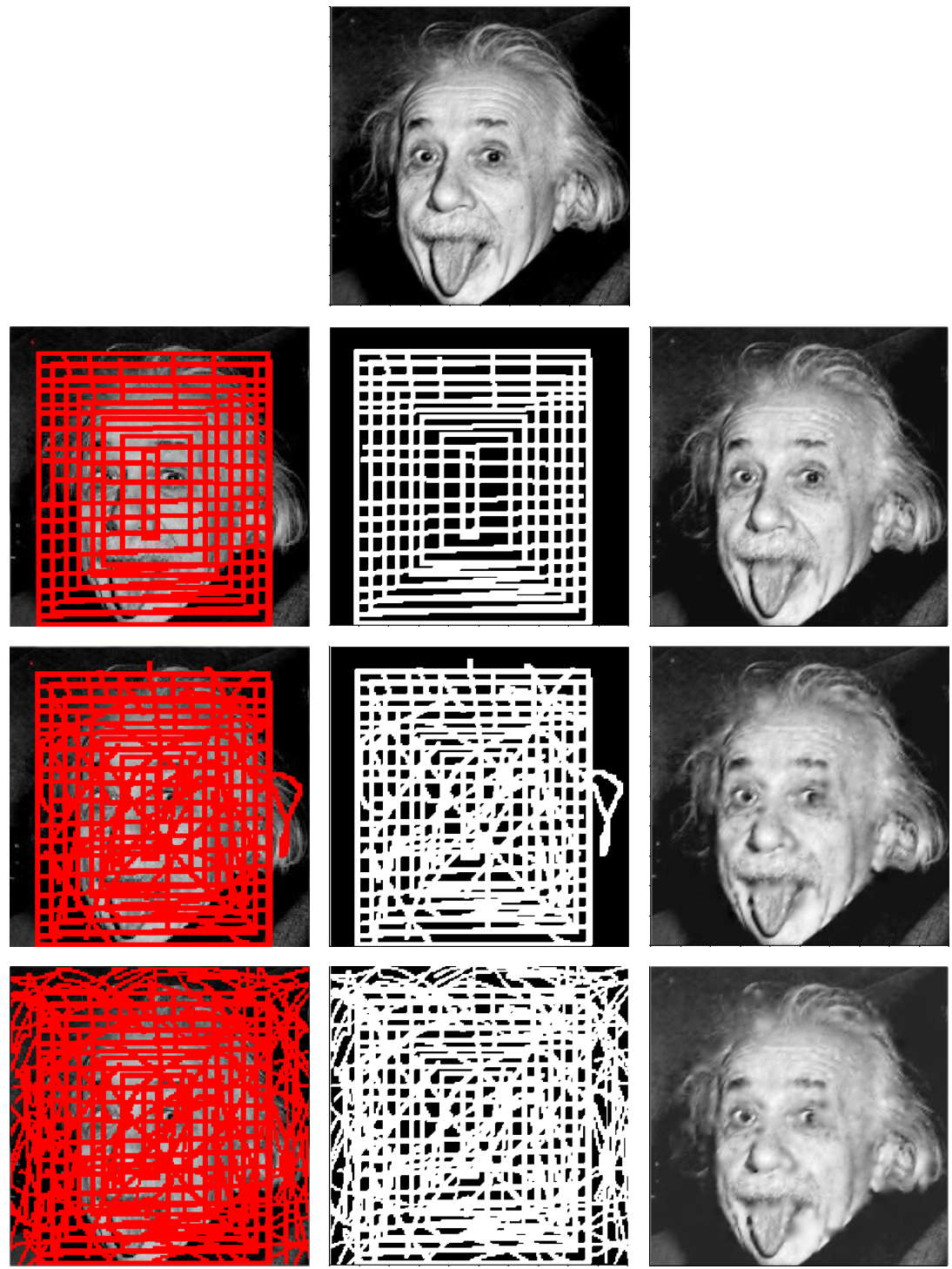

FiguRE 6. The damaged, mask and restored images, respectively. Over: $45 \%$ of pixels are damaged- Middle: $55 \%$ of pixels are damaged. Below: $75 \%$ of pixels are damaged.

[23] J. Cahn and E. Hilliard, Free energy of a nonuniform system. I. Interfacial free energy, Journal of Chemical Physics, $\mathbf{2 8}$.

[24] M. Carriero, A. Leaci and F. Tomarelli, Free gradient discontinuity and image inpainting, Journal of Mathematical Sciences, 161 (2012), 805-819.

[25] A. Chambolle and B. Bourdin, Implementation of an adaptive finite-element approximation of the Mumford-Shah functional, Numer. Math., 85 (2000), 609-646.

[26] A. Chambolle and G. D. Maso, Discrete approximation of the Mumford-Shah functional in dimension two, M2AN Math. Model. Numer. Anal., 33 (1999), 651-672.

[27] T. Chan and J. Shen, Mathematical models for local non-texture inpainting, SIAM Journal on Applied Mathematics, 62 (2002), 1019-1043. 

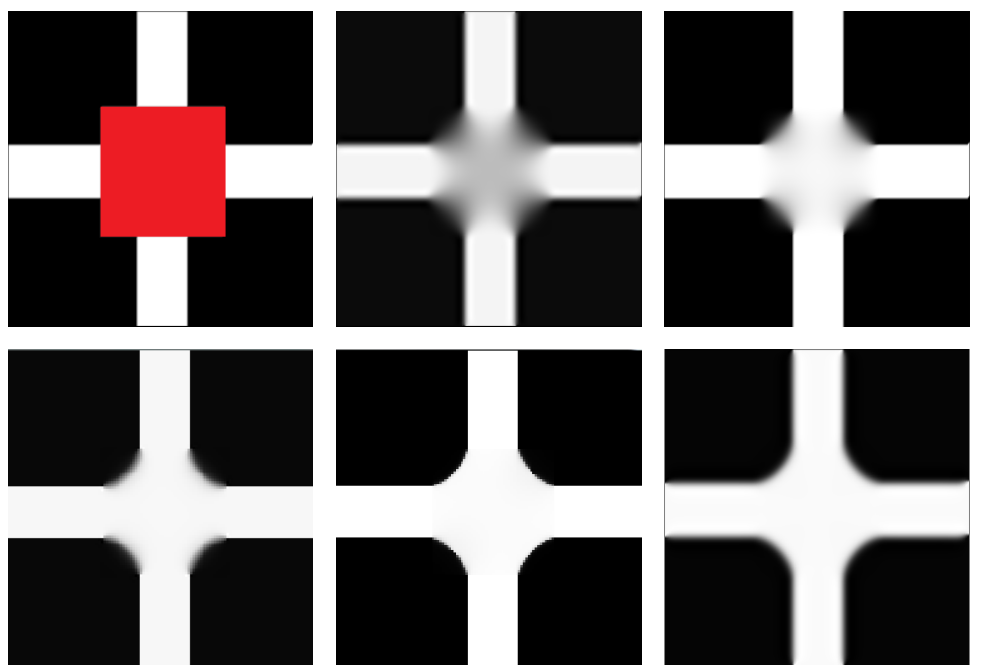

FiguRE 7. Top row: Damaged and restored images using model (4) and adaption (iterations 1 and 5). Bottom row: Restored images using model (4) and adaption (iterations 10 and 20) and CahnHilliard model.
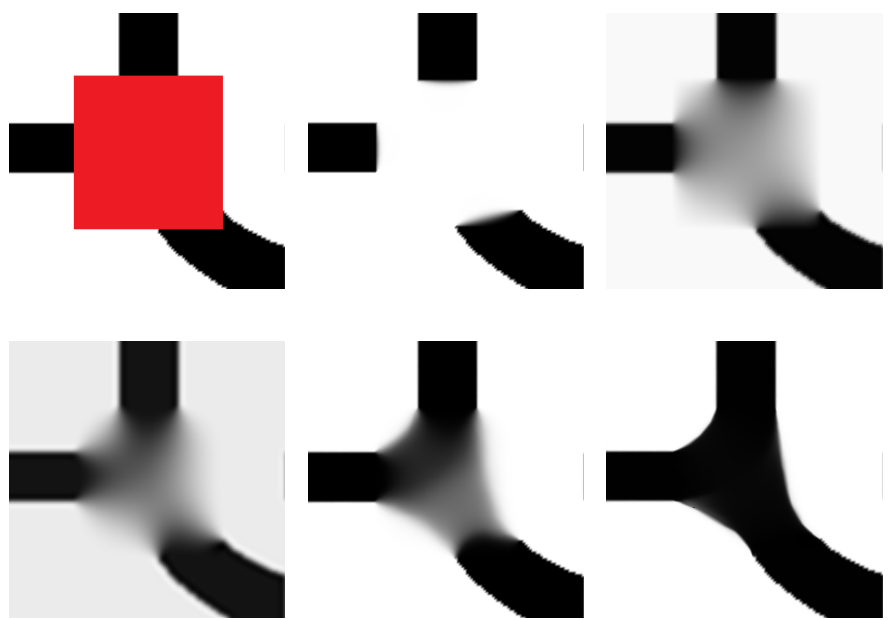

Figure 8. Top row: Damaged image and restored ones using total variation and harmonic models, respectively. Bottom row: Restored images using biharmonic model and our approach at iterations 5 and 20 , respectively.

[28] L. Cherfils, H. Fakih and A. Miranville, A CahnHilliard System with a Fidelity Term for Color Image Inpainting, Journal of Mathematical Imaging and Vision, 1-15.

[29] L. Cherfils, H. Fakih and A. Miranville, Finite-dimensional attractors for the BertozziEsedoglu-Gillette-Cahn-Hilliard, Inverse Problems and Imaging, 9 (2015), 105-125.

[30] G. Chung, J. Hahn and X. Tai, A fast algorithm for Euler's elastica model using augmented Lagrangian method, SIAM Journal on Imaging Sciences, 4 (2011), 313-344.

[31] A. Efros and Leung, Texture synthesis by non-parametric sampling, in Proceedings of the IEEE Conference on CVPR, vol. 2 of ICCV'99, IEEE Computer Society, 1999. 
[32] S. Esedoglu and J. Shen, Digital image inpainting by Mumford-Shah-Euler model, European Journal of Applied Mathematics, 13 (2002), 353-370.

[33] L. C. Evans, Partial Differential Equations, vol. 19 of Graduate Studies in Mathematics, American Mathematical Society, Providence, Rhode Island, 1998

[34] S. Geman and D. Geman, Stochastic relaxation, Gibbs distributions, and the Bayesian restoration of images, IEEE Transactions on Pattern Analysis and Machine Intelligence, 6 (1984), $721-741$.

[35] F. Hecht, New development in freefem++, Journal of Numerical Mathematics, 20 (2002), 251-265.

[36] M. Kallel, M. Moakher and A. Theljani, The Cauchy problem for a nonlinear elliptic equation: Nash-game approach and application to image inpainting, Inverse Problems and Imaging, $\mathbf{9}$ (2015), 853-874.

[37] S. Masnou, Filtrage et désocclusion d'images par méthodes d'ensembles de niveau, PhD thesis, Université Paris-Dauphine, 1998.

[38] S. Masnou, Disocclusion a variational approach using level lines, IEEE Transactions on Image Processing, 11 (2002), 68-67.

[39] S. Masnou and J. M. Morel, Level-lines based disocculusion, Proceedings of 5th IEEE International Conference on Image Processing, 259-264.

[40] D. Mumford and J. Shah, Optimal approximations by piecewise smooth functions and associated variational problems, Communications on Pure and Applied Mathematics, 42 (1989), $577-685$.

[41] M. Nitzberg, D. Mumford and T. Shiota, Filtering, segmentation and depth, vol. 662 of Lecture Notes in Computer Science. Springer.

[42] J. Shen, S. H. Kang and T. Chan, Euler's elastica and curvature-based inpainting, SIAM J. Imaging Sci., 63 (2002), 564-592.

[43] M. Zanetti and A. Vitti, The Blake-Zisserman model for digital surface models segmentation, ISPRS Annals of Photogrammetry, Remote Sensing and Spatial Information Sciences, vol. $I I-5 / W 2,355-360$.

Received $\mathrm{xxxx} 20 \mathrm{xx}$; revised $\mathrm{xxxx} 20 \mathrm{xx}$.

E-mail adresses: thaljanianis@gmail.com, zakaria.belhachmi@uha.fr, moez.kallel@ipeit.rnu.tn, maher.moakher@enit.rnu.tn 